\title{
Control de calidad de fitofármacos con la utilización de Resonancia Magnética Nuclear (RMN) y la aplicación del análisis de componentes principales (PCA)
}

\author{
Quality control of phytopharmaceuticals with the \\ use of Nuclear Magnetic Resonance (NMR) and the \\ application of principal component analysis (PCA)
}

Javier Quesada-Espinoza', Renato Murillo-Masís²

Fecha de recepción: 27 de enero de 2019 Fecha de aceptación: 26 de abril de 2019

Quesada-Espinoza, J; Murillo-Masís, R. Control de calidad de fitofármacos con la utilización de Resonancia Magnética Nuclear (RMN) y la aplicación del análisis de componentes principales (PCA). Tecnología en Marcha. Vol. 32-4. OctubreDiciembre 2019. Pág 81-94.

doi) https://doi.org/10.18845/tm.v32i4.4794 


\title{
Palabras clave
}

Fitofármacos; Gingko biloba; Hypericum perforatum; Silybum marianum; Equinacea; Resonancia Magnética Nuclear; Componentes Principales; Control de calidad.

\section{Resumen}

Los fitofármacos, aunque generalmente están compuestos de productos naturales, deben ser estrictamente controlados en cuando a su contenido de principios activos. Existen varios métodos de control de calidad entre los que se destacan la cromatografía líquida y la espectrometría de masas; en nuestra investigación llevamos a cabo un control de calidad con la utilización de Resonancia Magnética Nuclear, método el cual es de fácil utilización e interpretación, y altamente versátil, además de ser una técnica no destructiva, y consecuentemente un estudio de componentes principales para obtener una mejor visualización de los resultados. Lo que se logró demostrar es que en Costa Rica existe una gran cantidad de productos comercializados que aparentemente no contienen los principios activos que deberían tener de acuerdo al etiquetado, como pudo demostrarse en el caso de los productos de Gingko biloba, Hypericum perforatum y Silybum marianum. Para el caso de Equinacea spp, el problema de la comparación es que los diferentes productos contienen varias especies del mismo género y esto dificulta un análisis detallado. Además, el análisis de los componentes principales hizo posible demostrar que es difícil utilizar esta técnica sin un conocimiento previo de las técnicas de análisis utilizadas y qué debe interpretarse. Los resultados también indican que las regulaciones en Costa Rica no son lo suficientemente rigurosas para este tipo de productos, y estas deben ser revisadas por la Agencia Nacional Reguladora.

\section{Keywords}

Phytopharmaceuticals; Gingko biloba; Hypericum perforatum; Silybum marianum; Echinacea; Nuclear Magnetic Resonance; Principal Components; Quality control.

\begin{abstract}
The phytopharmaceuticals, although they are generally composed of natural products, must be strictly controlled regarding their content of active ingredients. There are several methods of quality control among which stand out liquid chromatography and mass spectrometry; in our research, we carried out a quality control with the use of Nuclear Magnetic Resonance, which is easy to use and interpret, and highly versatile, as well as non-destructive technique, and with this information a study of principal components was carried out in order to obtain a better visualization of the results. In this work we demonstrated that in Costa Rica there is a large number of products marketed that apparently do not contain the active ingredients that should have according to the labeling, as could be demonstrated in the case of the products of Gingko biloba, Hypericum perforatum and Silybum marianum. In the case of Equinacea spp, the problem of the comparison was that the different products contain several species of the same genus and this makes a detailed analysis difficult. In addition, the analysis of main components made it possible to demonstrate that it is difficult to use this technique without prior knowledge of the analysis techniques used, and what must to be interpreted. The results also indicate that regulations in Costa Rica are not rigorous enough for this type of products, and these should be reviewed by the National Regulatory Agency.
\end{abstract}




\section{Introducción}

\section{Regulación}

Existe una gran cantidad de plantas medicinales que tienen un uso muy sólido en las sociedades, tanto que actualmente se comercializan en forma de cápsulas, pastillas, jarabes, gránulos y otras formas. Estos productos han tenido en el público en general una gran aceptación por el sentimiento, erróneo en muchos casos, que al ser productos naturales tienen menos efectos secundarios y son mejores que los medicamentos sintéticos.

El mercado de este tipo de productos, llamados en muchos casos nutracéuticos o fitofármacos, ha tenido un crecimiento anual desde el 2000 de 5\% y se estimó que globalmente era de \$30 billones para ese año [1] y para el año 2018 fue de $\$ 126.3$ billones [2]. De acuerdo a Health Canada [3] [4] se define un nutracéutico como "un producto que es aislado de un alimento y tiene un beneficio en la salud o prevención de enfermedades crónicas". Mientras que un fitofármaco se define como "un fármaco de origen vegetal" [5].

Todos estos productos, al tener sustancias activas que pueden afectar el estado de salud de un organismo deben regularse de forma adecuada, de manera que, al igual que los medicamentos, se logre demostrar que el producto que está en el mercado es de calidad, seguro y tiene la eficacia para cual se pretende su uso.

En lo que respecta a regulación de estos productos, la FDA (Administración de Medicamentos y Alimentos de los Estados Unidos) aclara que "Estos productos no están destinados a diagnosticar, tratar, curar o prevenir ninguna enfermedad". Sin embargo, en Canadá, nutracéuticos y suplementos dietéticos se consideran entre los "productos de salud naturales" regulados y autorizados por la Dirección de Productos de Salud Natural y sin Prescripción desde el 2004 [6]. En el caso de la Unión Europea no existía una regulación precisa hasta 1997, donde se inició la creación de una regulación que entró en vigor en el 2002 [7]. En esta regulación el punto principal es la seguridad, además de que estos productos deben demostrar que contienen los principios activos que indican, en las cantidades adecuadas.

En lo que respecta a Costa Rica los productos naturales están regulados por el RTCA 11.03.64:11 [8] "Productos Farmacéuticos Productos Naturales Medicinales para Uso Humano, Requisitos de Registro Sanitario". En este caso, en lo referente a la identidad del producto se debe presentar una fórmula cualitativa y cuantitativa del producto, pero no se especifican claramente aspectos de los principios activos que deben contener los productos naturales.

\section{Control de calidad}

Para controlar la calidad de estos productos generalmente se requieren métodos de cromatografía líquida (HPLC) acoplado a espectrometría de masas para poder identificar los diferentes componentes que le proporcionan la actividad al producto, y, además, estos deben cuantificarse. [9] [10] [11].

A pesar de esto, existen métodos más sencillos y que requieren menos tiempo, con los que se puede hacer un control de calidad de estos productos de manera rutinaria, y que, además, sean de más sencilla visualización [12]. En este estudio, se lleva a cabo una medición de espectros de ${ }^{1} \mathrm{H}-\mathrm{NMR}$ de muestras de varios productos naturales (Echinacea purpurea, Silybum marianum, Gingko biloba, e Hypericum perforatum ) tomando como referencia productos obtenidos de mercados altamente regulados. Con los espectros obtenidos se llevó a cabo un estudio de componentes principales, para, de una manera gráfica, poder demostrar la calidad de los productos basados en estándares de referencia. 
Aunque la resonancia magnética nuclear no es un método muy sensible, como la cromatografía líquida y la espectrometría de masas, es una técnica muy reproducible, no destructiva, es de preparación sencilla y de fácil aplicación.

\section{Fitofármacos y nutracéuticos en Costa Rica}

Dentro de los fitofármacos más vendidos en Costa Rica están la Echinacea (Echinacea purpurea), el Milk thistle -cardo mariano- (Silybum marianum), la hierba de San Juan (Hypericum perforatum) y el Gingko biloba.

El G. biloba (Ginkgoaceae) es un árbol pequeño nativo de China, pero extensamente plantado como ornamental. Los extractos estandarizados de las hojas se comercializan contra enfermedad vascular y demencia del adulto mayor. Se ha demostrado que los extractos mejoran la circulación cerebrovascular ayudando a evitar la disminución de la función cognitiva y los procesos de memoria. En la edad avanzada el ginkgo puede ejercer efectos benéficos mejorando esta circulación y ayudando con otros síntomas como el vértigo, el tinitus y pérdida de audición. [13].

Los constituyentes activos se han caracterizado como mezclas de terpenos y flavonoides. Las hojas secas contienen 0,1 - 0,25\% de lactonas terpénicas, que comprenden cinco ginkgólidos (A, B, C, J, y M) y un bilobálido. El bilobálido comprende aproximadamente 30-40\% de la mezcla, mientras que la ginkgólido A es el ginkgólido predominante (aproximadamente 30\%). Los ginkgólidos son diterpenoides en naturaleza, mientras que el bilobalido se describe como sesquiterpenoide. El contenido de flavonoides de las hojas secas es de 0,5-1,0\%, y consiste en una mezcla de mono-, di- y triglicósidos de los flavonoles kaempferol y quercetina, y algunos biflavonoides. Los extractos de ginkgo para el consumo suelen estandarizarse para contener glucósidos de flavonoides y lactonas terpénicas en una proporción de 24\% a 6\%, o 27\% a 7\%. [14] [15].

La Silybum marianum (Asteraceae) (cardo de leche) es una planta que se encuentra comúnmente en el área mediterránea de Europa. Las semillas producen 1,5-3\% de flavonolignanos. Colectivamente denominado silimarina. Esta mezcla contiene principalmente silibina, junto con siliristina, silidianina y pequeñas cantidades de isosilibina. Tanto la silibina como la isosilina son mezclas equimolares de dos diastereoisómeros trans. La S. marianum es ampliamente utilizada en la medicina tradicional europea; las frutas se utilizan para tratar una variedad de trastornos hepáticos. Se ha demostrado que la silimarina protege el hígado de animales contra los efectos dañinos del tetracloruro de carbono, la tioacetamida, fármacos tales como paracetamol y algunas las toxinas como la $\alpha$-amanitina. La silimarina se puede utilizar en muchos casos de enfermedad hepática y lesión, aunque sigue siendo de uso secundario en la medicina convencional. Estos compuestos parecen tener dos modos principales de acción. Actúan sobre la membrana celular de los hepatocitos inhibiendo la absorción de toxinas y en segundo lugar, debido a su naturaleza fenólica, pueden actuar como antioxidantes y eliminadores de radicales libres que causan daño hepático. [16], [17]

La Echinacea purpurea (Asteraceae) es una planta medicinal utilizada como inmunoestimulante y particularmente como profiláctico y tratamiento del resfriado común. Su actividad proviene de una serie de alquilamidas, amidas de ácidos poliinsaturados con isobutilamina. Estas son predominantemente dieno-diinos de 11 y 12 carbonos. También se encuentran presentes derivados del ácido cafeico, cómo el ácido chicórico. La actividad en realidad no puede ser adjudicada a ninguna sustancia específica, [18]. Otras plantas del mismo género como la $E$. angustifolia poseen propiedades similares, y también se encuentra presente en los preparados de Equinaceae. 
Las flores secas de la hierba de San Juan (Hypericum perforatum, Guttiferae / Hypericaceae) se han utilizado como una planta medicinal y se ha empleado por sus propiedades antisépticas y cicatrizantes. El principal cultivo es comercializado como un antidepresivo, que se dice que es tan eficaz en su acción como los antidepresivos ampliamente prescritos.

Existe evidencia clínica considerable de que los extractos de hierba de San Juan son eficaces en el tratamiento de la depresión leve a moderada y el estado anímico.

La hierba seca contiene cantidades significativas de derivados fenólicos, incluyendo 4-5\% de flavonoides, aunque se considera que la actividad antidepresiva se deriva principalmente de las estructuras de naftodiantronas tales como hipericina (aproximadamente $0.1 \%$ ) y pseudohipericina (aproximadamente 0.2\%), y la hiperforina. La planta fresca también contiene niveles significativos de protohipericina y protopseudohipericina, que se convierten en hipericina y pseudohipericina durante el secado y el procesamiento, como resultado de la irradiación con luz visible. [19]

En este estudio se lleva a cabo un control de calidad de los fitofármacos previamente mencionados con la utilización de ${ }^{1} \mathrm{H}-\mathrm{RMN}$ los cuales se comercializan en Costa Rica. Conociendo los principios activos de cada uno de ellos, se realizó una comparación de un producto de un mercado altamente regulado con productos locales, y se efectuó un estudio de componentes principales (PCA) para poder presentar los datos de la comparación de una manera comprensible.

\section{Procedimiento}

\section{Reactivos}

Se empleó éter metil ter-butílico, metanol y cloroformo marca Sigma-Aldrich o Fluka. Cloroformo deuterado $\left(\mathrm{CDCl}_{3}\right)$, dimetilsolfóxido deuterado $\left(\mathrm{DMSOd}_{6}\right)$ y metanol deuterado $\left(\mathrm{CD}_{3} \mathrm{OD}\right)$ marca Sigma-Aldrich.

\section{Adquisición de muestras}

Se adquirieron cápsulas elaboradas con base en Hipericum perforatum, Silybum marianum, Equinacea spp y Ginkgo biloba. Se compraron muestras de una marca comercial aprobada en un mercado altamente regulado que certifica el contenido de compuestos a los cuales se atribuye propiedades medicamentosas. Esta se empleó como material de referencia. Por cada planta se adquirieron, además, cinco muestras de diferentes marcas locales en América Latina (M1, M2, M3, M4 y M5). Ninguna de ellas certificaba el contenido de compuestos activos. Algunas especificaban el contenido de planta por cápsula.

\section{Preparación de las muestras para NMR}

De cada producto se tomaron tres cápsulas o el equivalente correspondiente al contenido reportado por la casa comercial reconocida. Se abrieron y al contenido finamente molido se extrajo con $20 \mathrm{~mL}$ del metanol los productos de H. perforatum, S. marianum y G. biloba y con éter metil ter-butílico los productos de Equinacea spp. (en algunos casos no se aclaraba la especie en cuestión). Se extrajo durante 20 min en un baño ultrasónico. Se filtró y se secó al vacío a una temperatura de $40^{\circ} \mathrm{C}$. El extracto seco de $S$. marianum se disolvió en $5 \mathrm{~mL}$ de una mezcla de cloroformo-metanol (1:1), los de Equinacea sp. se disolvieron en cloroformo y en metanol el extracto de $G$. biloba y $H$. perforatum. La mitad del volumen anterior fue colocada en un balón y secada. Los extractos de $S$. marianum y el de G. biloba fueron redisueltos en una mezcla $\mathrm{CDCl}_{3} / \mathrm{CD}_{3} \mathrm{OD}(9: 1)$. Los extractos secos de Echinacea spp. fueron redisueltos en $\mathrm{CDCl}_{3}$ y el extracto de $H$. perforatum se redisolvió en $\mathrm{DMSOd}_{6}$. Un volumen de 0,5 $\mathrm{mL}$ de cada uno fue transferido a un tubo para muestras de NMR de $5 \mathrm{~mm}$ ID. 


\section{Mediciones de NMR}

Todos los espectros fueron medidos en un equipo de NMR marca Bruker Ascend de 600 $\mathrm{MHz}$, operando en modo protónico a una frecuencia de $600,13 \mathrm{MHz}$. Para cada espectro se adquirieron 128 escaneos, con datos colectados dentro de $64 \mathrm{~K}$ puntos de datos, con un ancho espectral de 20,55 ppm, un ángulo de pulso de $30^{\circ}$, un retraso de relajamiento de $1 \mathrm{~s}$ y un tiempo de adquisición de 2,66 s. A los FIDs se les aplicó un LB = 0,3 antes de la transformación de Fourier.

\section{Reducción y pretratamiento de datos de NMR}

Todos los espectros de ${ }^{1} \mathrm{H}-\mathrm{NMR}$ fueron apodizados, corregida la fase y la línea base, referenciados con respecto a la señal a 7,26 ppm del $\mathrm{CDCl}_{3} 0$ 2,49 ppm del $\mathrm{DMSOd}_{6}$ y se alinearon las regiones de los diferentes espectros. Se eliminaron regiones del espectro dependiendo del producto estudiado y las señalas características que presenta:S. marianum: se trabajó la región de 5,70 ppm a 7,20 ppm; Equinacea sp.: se trabajó la región de 5,50ppm a 7,20 ppm; G. biloba: se trabajó la región de 5,50 ppm a 8,20 ppm. Se sustrajo la región del disolvente (7,20 ppm a 7,30 ppm); H. perforatum: se trabajó la región de 5,80 ppm a 7,10 ppm.

Las regiones seleccionadas fueron reducidas a "buckets" espectrales de 0,02 ppm para el análisis de componentes principales. Todo lo anterior se realizó empleando el software MestReNova versión 10.0.2 (Mestrelab Research S.L.)

Análisis multivariado de los datos

Se realizó un autoescalado, normalización por área total a 1,0 y centrado de datos utilizando la media y el escalamiento se realizó ustilizando Paretto el cual fue efectuado antes de realizar un análisis de componentes principales (PCA). Se aplicó un método de reconocimiento de patrones no supervisado para examinar la variación intrínseca de los datos empleando el programa Solo versión 8.0.1 (Eigenvector Research, Inc).

\section{Resultados y discusión}

Las plantas medicinales estudiadas, como se ha mencionado, presentan compuestos con estructuras químicas muy particulares de cada especie. Estas estructuras tienen espectros de ${ }^{1} \mathrm{H}-\mathrm{RMN}$ que las hacen fácilmente distinguibles. Con el propósito de poder hacer un estudio comparativo de las muestras en el mercado en cuanto a su contenido de principios activos, se llevaron a cabo extracciones de cantidades iguales -según la dosis recomendada en la etiqueta de los productos- de los diferentes productos que contienen las plantas medicinales que se lograron obtener en el mercado local, y se midieron espectros ${ }^{1} \mathrm{H}-\mathrm{RMN}$ de cada uno de ellos -todos en las mismas condiciones-. Además, se tomó un producto de una marca de venta en USA, considerada un mercado altamente regulado. Se analizaron 6 marcas incluyendo la marca vendida en USA. Luego de haber hecho varios extractos con el fin de optimizar los disolventes se pudo verificar que los principios activos se han extraído eficientemente.

La Echinacea angustifolia contiene una serie de alquilamidas diénicas-diínicas y ácido chicórico como marcadores y posibles compuestos activos. Las señales de los dobles enlaces conjugados son claramente distinguibles entre 5.70 y $7.30 \mathrm{ppm}$ en el espectro ${ }^{1} \mathrm{H}-\mathrm{RMN}$ [20], la figura 1a presenta el espectro ${ }^{1} \mathrm{H}-\mathrm{RMN}$ (fragmento) del producto de referencia donde estos componentes son claramente distinguibles.

En la figura 1b se compara el producto de referencia con los diferentes productos locales. Como puede observarse, existe una gran diferencia en los espectros de los productos estudiados. Una posible explicación de la diferencia encontrada entre los diferentes productos puede ser que 
cuando se habla de Echinacae existen varias especies, dentro de las que se pueden mencionar la E. purpurea, la E. pallida y la E. angustifolia [21], y posiblemente las especies utilizadas son diferentes en los productos. En el caso del producto de USA se trata de $80 \%$ de E. purpurea y $20 \%$ de E. angustifolia. Sin embargo, un común denominador que es necesario, es la presencia de los principios activos a los que se les adjudica la actividad inmunoestimulante.

a

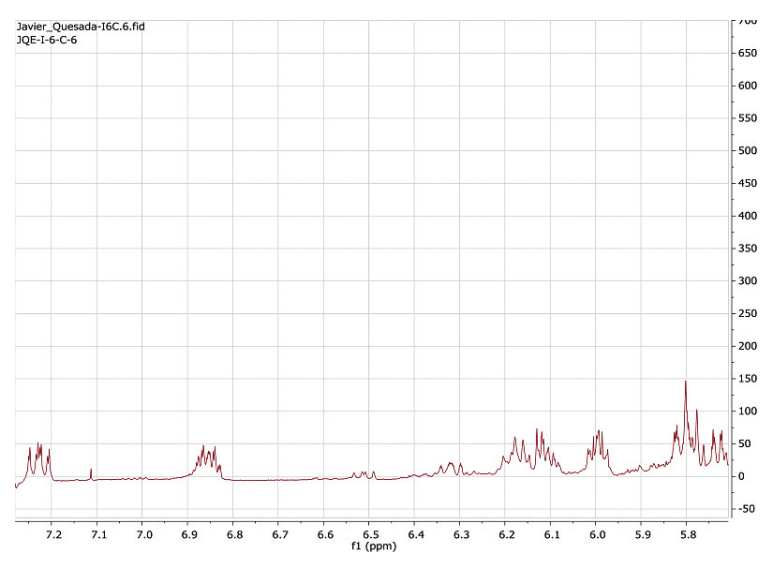

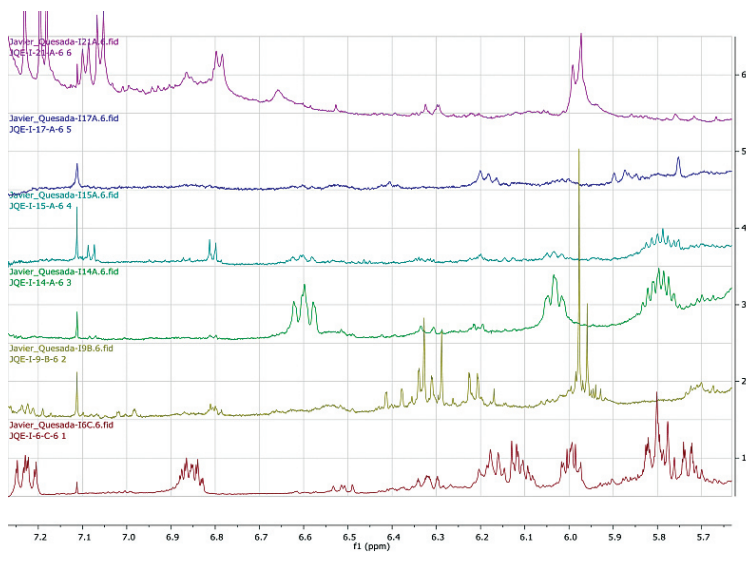

b

Figura 1. a) Espectro ${ }^{1} \mathrm{H}-\mathrm{RMN}$ del extracto de Echinacae purpurea (muestra de USA) 5.70 a 7.30 ppm. $\left(\mathrm{CDCl}_{3}, 600\right.$ $\mathrm{MHz})$. b) Espectros representativos de las muestras de Echinacae de los productos locales, $\left(\mathrm{CDCl}_{3}, 600 \mathrm{MHz}\right), \mathrm{el}$ espectro del producto de USA corresponde al espectro 1 (parte de abajo).

El estudio de componentes principales (PC) sugiere que no todas las muestras son diferentes como se aparenta al observar los espectros de ${ }^{1} \mathrm{H}-\mathrm{RMN}$. Existen tres marcas comerciales que se asemejan a la empleada como referencia y que otras dos no, pero estas no se parecen entre sí. Este comportamiento se observa al considerar la región del espectro ${ }^{1} \mathrm{H}-\mathrm{RMN}$ entre 5.50 y 7.20 ppm.

En la figura 2a se representan los dos primeros componentes (PC1 vs PC2) -que cubren alrededor del 70\% de los datos del espectro-, se puede observar que PC1 separa la muestra M5 de las demás muestras y la referencia, mientras PC2 lo hace para la muestra M1. El gráfico de loading correspondiente -figura 3- (que considera el peso o efecto de las señales sobre el estudio de componentes principales) permite identificar la correlación existente entre las variables originales y los factores o componentes obtenidos en el análisis. En este caso, para la componente 1 explica que las regiones de 5.80 ppm y 7.20 son importantes en la diferenciación de M5 del resto de las preparaciones comerciales de Echinacea. La figura 2b. muestra que las regiones alrededor de 5.90 y 6.30 ppm diferencia a la preparación M1 de las demás. Si la representación se realiza con PC1 vs PC3 (62\% de los datos), la separación de las muestras es diferente. En este caso la muestra 2 se separa del resto y la muestra 1 no lo hace, como se muestra en la figura 2b. El gráfico de loading (figura 3b.) muestra como las señales en la región de 6.00 ppm y 6.57 ppm son las que provocan la separación de la muestra 2 de las demás en una especie de intercambio con la muestra M1. Las señales de los dobles enlaces conjugados son las que producen el cambio indicado anteriormente. 


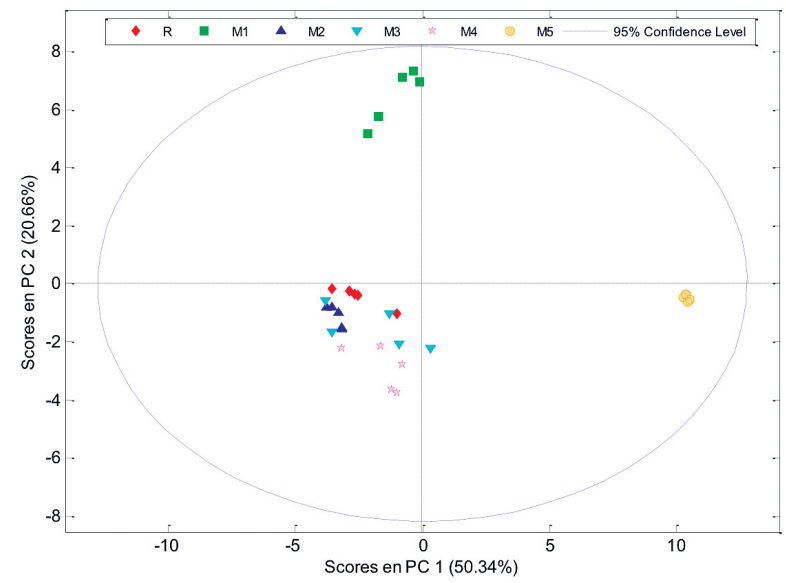

a.

Figura 2. a) Gráfico de score PC1 vs PC2 derivado de los espectros de ${ }^{1} \mathrm{H}-\mathrm{RMN} 600 \mathrm{MHz}$ de los extractos de Echinacea. b) Gráfico de score PC1 vs PC3 derivado de los espectros de ${ }^{1} \mathrm{H}-\mathrm{RMN} 600 \mathrm{MHz}$ de los extractos de Echinacea. En ambos gráficos se pobservan 5 puntos de cada color, lo que significa que de cada marca se realizaron 5 réplicas. a.

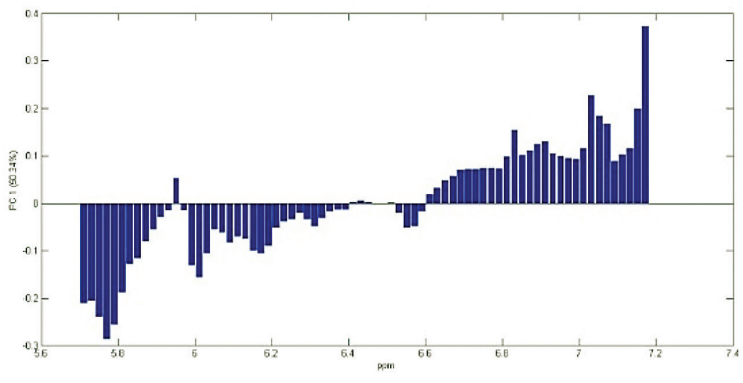

Figura 3. a) Gráfico de loading para la PC1 derivada de los espectros de $1 \mathrm{H}-\mathrm{RMN} 600 \mathrm{MHz}$ de los extractos de Echinacea. b) Gráfico de loading para la PC2 derivada de los espectros de $1 \mathrm{H}-\mathrm{RMN} 600 \mathrm{MHz}$ de los extractos de Echinacea.

\section{Silybum marianum}

Para el caso de $S$. marianum es bien conocido que los flavonolignanos son los componentes activos de la planta y las señales de estos compuestos son claramente distinguibles en el espectro de ${ }^{1} \mathrm{H}-\mathrm{RMN}$. La figura 4 a presenta el espectro ${ }^{1} \mathrm{H}-\mathrm{RMN}$ del producto tomado como referencia -se muestra el ámbito de 4.3 a 7.2 ppm, donde presentan señales los componentes activos-, a saber, la silimarina.

Observando los espectros de los productos locales -presentados de manera comparativa con el producto de referencia en la figura 4, se puede observar una señal pronunciada a 5.30 ppm, típica de ácidos grasos insaturados presentes en plantas. Esta señal no se encuentra presente en el producto de referencia. Esto puede significar que las muestras locales contienen en sus extractos una alta cantidad de ácidos grasos. Esto por supuesto, va en detrimento de la cantidad de principio activo que se requiere en la cápsula para producir el efecto hepatoprotector del que se caracteriza la S. marianum. Esta observación podría llevar a la suposición que las muestras locales se encuentran adulteradas con otra planta con un alto contenido de ácidos grasos. 
a.
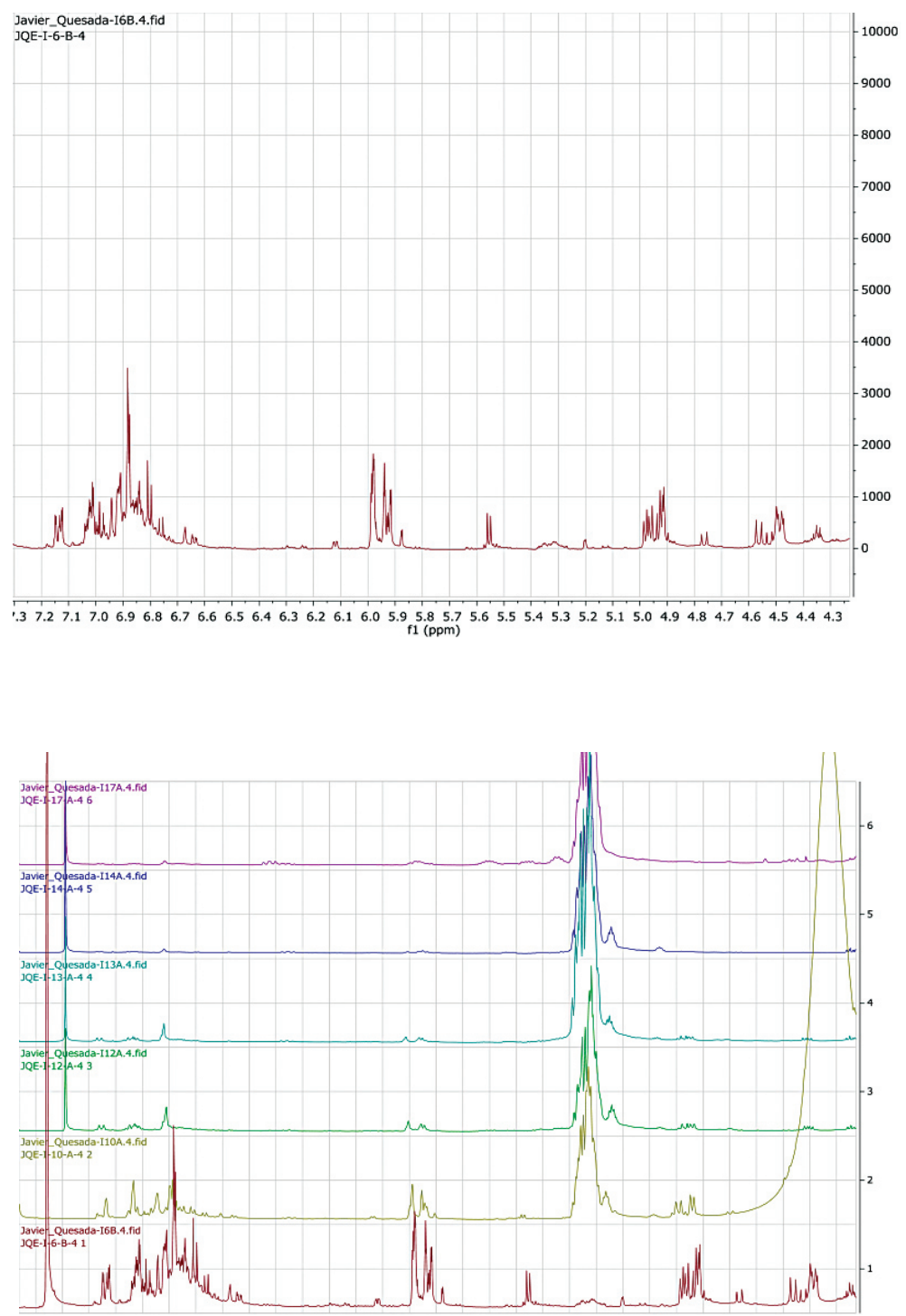

b.

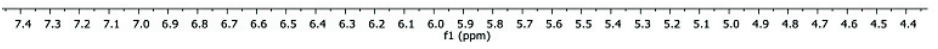

Figura 4. a) Espectro ${ }^{1} \mathrm{H}-\mathrm{RMN}$ del extracto de $S$. marianum (muestra de USA) 4.20 a $7.30 \mathrm{ppm}$. $\left(\mathrm{CDCl}_{3}-\mathrm{CD}_{3} \mathrm{OD}, 600\right.$ $\mathrm{MHz}$ ). b) Espectros ${ }^{1} \mathrm{H}-\mathrm{RMN}$ de muestras de diferentes productos de $S$. marianum comparadas con el espectro del producto de referencia.

Los productos indicados como 1-10-A, 1-12-A y 1-13-A (espectros 2, 3 y 4 de la figura 4b) presentan también señales de los compuestos activos, sin embargo, tanto en esos productos, como los otros dos contienen una cantidad considerable de ácidos grasos.

La figura 5 muestra el gráfico de scores del análisis de componentes principales empleando las regiones del espectro entre 5.70 y 7.20 (para minimizar el efecto de las señales de los ácidos grasos se excluye la región de los ácidos grasos de 5.30 ppm). Aquí se observa como la muestra referencia se encuentra separada espacialmente de las otras muestras de productos de marcas comercializadas en Costa Rica. Esto es consistente con las observaciones efectuadas a partir de los espectros de ${ }^{1} \mathrm{H}-\mathrm{RMN}$. Significa que si existen diferencias importantes en los productos debidas a los compuestos característicos de $S$. marianum que le brindan sus propiedades hepatoprotectoras. El componente 1 es el que muestra la separación y, además, acumula casi el $74 \%$ de la varianza, por esto el componente 3 no se considera. 


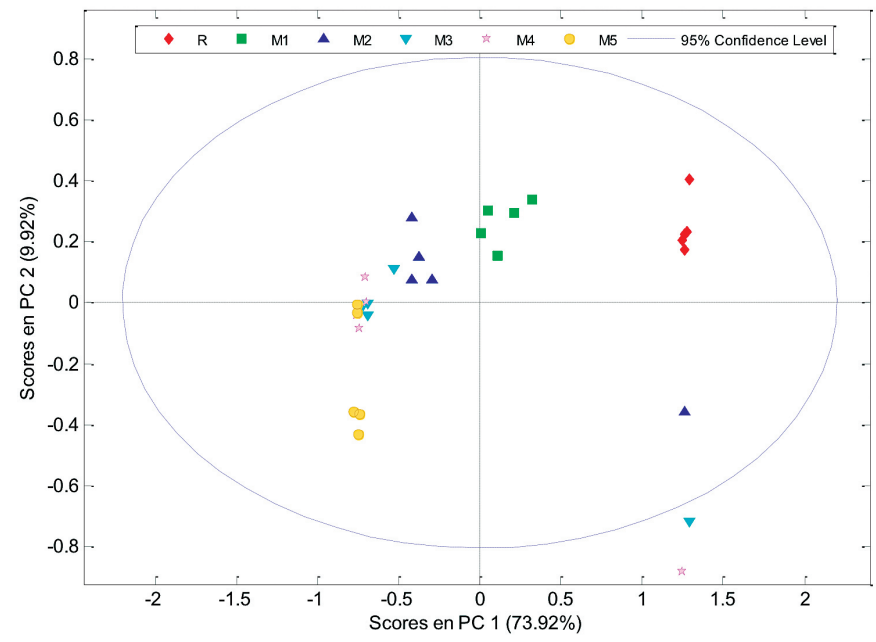

Figura 5. Gráfico de score PC1 vs PC2 derivado de los espectros de 1H-RMN $600 \mathrm{MHz}$ de los extractos de Silybum marianum.

En el gráfico de loading (no se muestra) para PC1 presenta la importancia de las señales entre 6.85 ppm y 6.91 ppm para diferenciar la muestra de referencia del resto de muestras de productos comerciales, las cuales se observan en la figura 5.

\section{Hipericum perforatum}

Los principios activos de la hierba de San Juan, son, como se discutió previamente, la hipericina y la hiperforina, sin embargo, derivados del ácido clorogénico y otros componentes, como varios flavonoides glicosilados, sirven como marcadores para la calidad de estos productos. Las señales de estos compuestos son claramente distinguibles en el espectro ${ }^{1} \mathrm{H}-\mathrm{RMN}$.

Es importante, por el tipo de compuestos presentes, comparar las regiones entre 6.00 ppm y $8.50 \mathrm{ppm}$ del espectro ${ }^{1} \mathrm{H}-\mathrm{RMN}$. En la figura 6 a se puede observar el espectro del extracto considerado como estándar, donde se observan las señales de los marcadores, de acuerdo a la literatura [22].

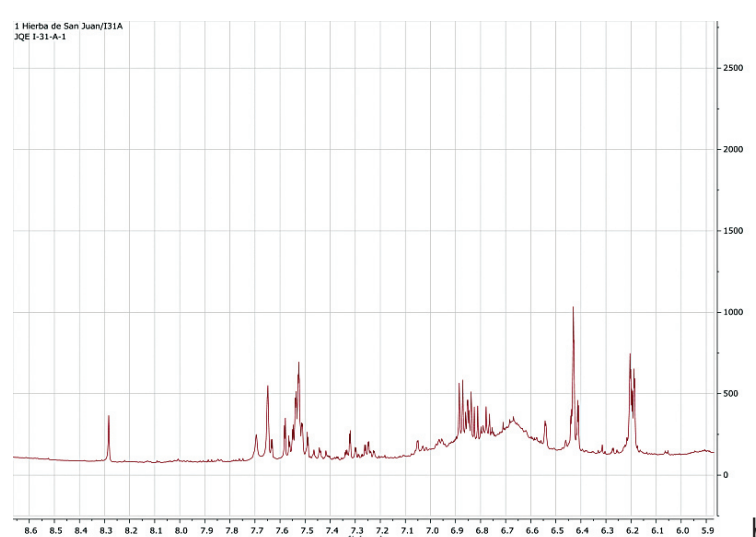

a.

Figura 6. a) Espectros ${ }^{1} \mathrm{H}-\mathrm{RMN}$ de $H$. perforatum. (DMSO d, $600 \mathrm{MHz}$ ), b) Espectros ${ }^{1} \mathrm{H}-\mathrm{RMN}$ de las muestras de H. perforatum (DMSO deuterado, $600 \mathrm{MHz}$ )

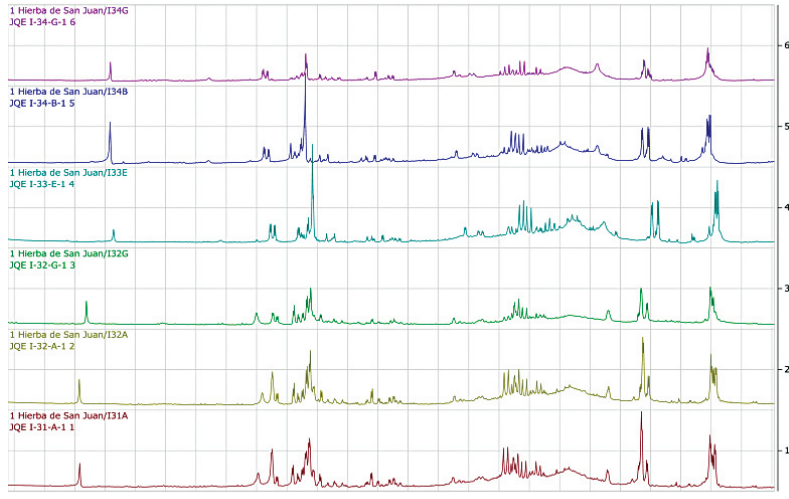

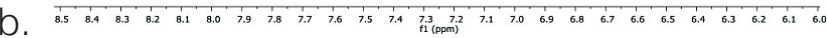


La figura 6 b muestra espectros de todas las muestras estudiadas, y lo que puede observarse claramente es los mismos grupos de señales en los mismos cambios químicos que el estándar, sin embargo, un análisis más cuidadoso de las señales demuestra diferencias apreciables en los tipos de compuestos y cantidades relativas. Por ejemplo, las señales entre 6.70 ppm y 6.80 ppm de las 4 muestras superiores presentan diferencias apreciables con respecto al patrón de comparación. La misma situación se observa con las señales entre 7.60 ppm y 7.70 ppm. En vista de que son tantos los marcadores que pueden dar señales en estas regiones del espectro, se puede plantear la hipótesis que las diferencias se deben a cantidades relativas diferentes en los componentes, lo cual lleva a la duda de que los diferentes productos tengan extractos estandarizados de hierba de San Juan.

Estas diferencias se constatan en el estudio de componentes principales (figura 7) en una clara separación entre la referencia, muestras 1 y 2 y las muestras 3,4 y 5. La componente 1 hace que se separen completamente. Según el gráfico de loading (no presentado) las señales a 6.05 ppm, 6.35 ppm, 6.60 ppm, 6.95 ppm y 7.08 ppm son las que producen la separación mencionada anteriormente.

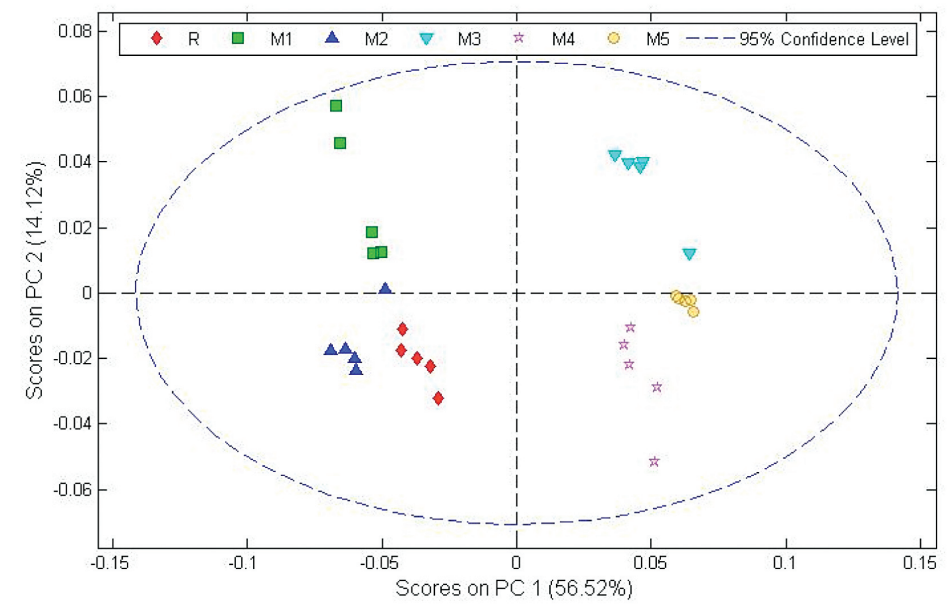

Figura 7. Gráfico de scores PC1 vs PC2 derivado de los espectros de ${ }^{1} \mathrm{H}-\mathrm{RMN} 600 \mathrm{MHz}$ de los extractos de $\mathrm{H}$. perforatum.

\section{Gingko biloba}

Finalmente, para el caso de G. biloba, tanto los ginkgólidos como los bilobálidos presentan señales en ${ }^{1} \mathrm{H}-\mathrm{RMN}$ características. [23]. Se obtuvo de la muestra de USA el espectro ${ }^{1} \mathrm{H}-\mathrm{RMN}$ de la figura 8. En este espectro se pueden observar las señales que indican la presencia de los principios activos de la planta medicinal.

Utilizando la misma metodología y estandarizando las alturas de los espectros, se presentan los datos para las muestras del producto de USA y los 5 productos regionales. Se observa claramente la presencia escasa o casi nula de los gingkólidos y los bilobálidos en los productos locales (figura 8b). Además, la presencia del kaemferol y la quercetina no pudo ser corroborada en los productos locales.

Al hacer el estudio de componentes principales para los diferentes productos que indican que contienen G. biloba, se hicieron varias modificaciones de las regiones del espectro seleccionadas 
que contienen las señales de los principios activos, sin embargo, no se logró una separación visual evidente con PC1 y PC2, lo que demuestra que el estudio de componentes principales no proporciona la información que fue evidente del espectro ${ }^{1} \mathrm{H}-\mathrm{RMN}$. En la figura 9 se presenta de manera ilustrativa los componentes PC1 y PC2 que juntos suman aproximadamente el 52\% de la varianza de los datos.

a.

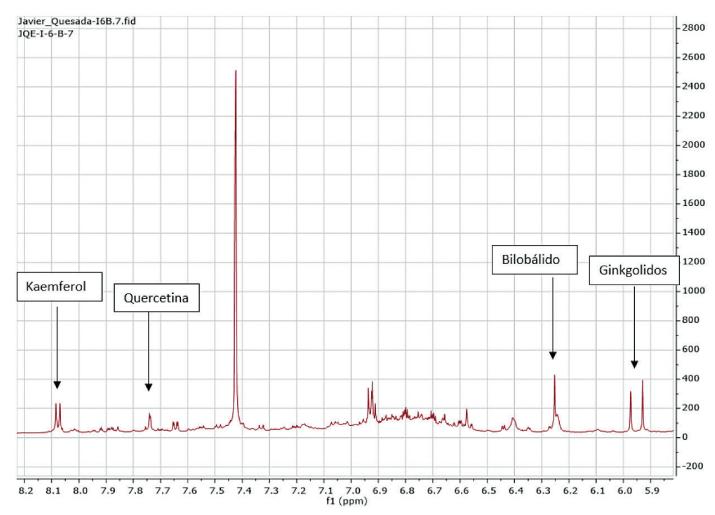

Figura 8. a) Espectro ${ }^{1} \mathrm{H}-\mathrm{RMN}$ del extracto de G. biloba marca de USA -una muestra-( $\left.\mathrm{CDCl}_{3}-\mathrm{CD}_{3} \mathrm{OD}, 600 \mathrm{MHz}\right)$. b) Espectros representativos de las muestras de ginkgo biloba de los productos locales, $\left(\mathrm{CDCl}_{3}-\mathrm{CD}_{3} \mathrm{OD}, 600 \mathrm{MHz}\right)$, el espectro del producto de USA corresponde al espectro 1 (parte de abajo).

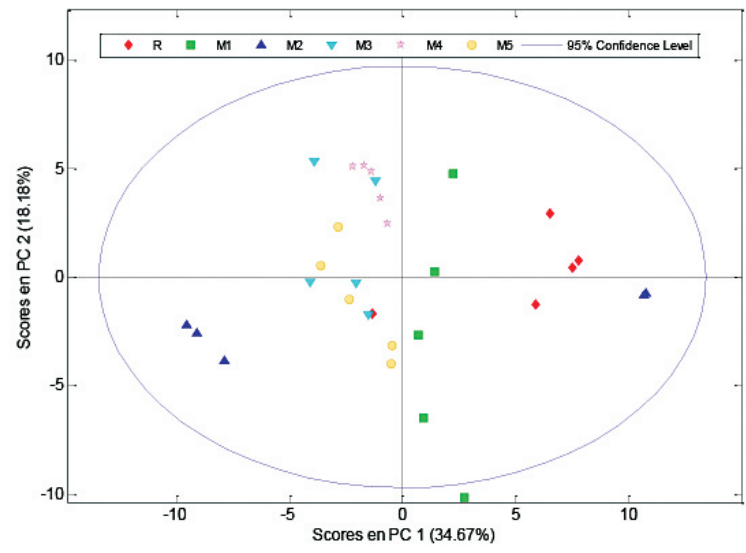

Figura 9. Gráfico de score PC1 vs PC3 derivado de los espectros de ${ }^{1} \mathrm{H}-\mathrm{RMN} 600 \mathrm{MHz}$ de los extractos de Ginkgo biloba.

El gráfico de "loading" producto del modelo generado a partir del empleo de los datos en la región de 5.50-8.20 ppm no presenta señal alguna que impacte la distribución observada en la figura 9, aspecto esperado porque no se aprecia separación.

\section{Conclusiones}

El control de calidad en los productos naturales medicinales que se encuentran en el mercado en los países en desarrollo es un tema de gran importancia, en especial, por el alto consumo que se da en estos países de este tipo de productos. La resonancia magnética nuclear, 
al ser uno de los métodos más veraces en la identificación de moléculas, resulta ser una herramienta de gran utilidad para dicho control. Este método resulta de fácil visualización, no destructivo y altamente versátil, aunque poco sensible. Para poder visualizar de manera relativamente cuantitativa los resultados de este análisis metabolómico, se realizó un estudio de componentes principales, donde, de una manera gráfica se puede apreciar que tan similares son unas muestras a otras con la utilización de ciertos parámetros. Esta metodología presenta, sin embargo, sus limitaciones, como pudo observarse en el caso de G. biloba, pero con una utilización correcta y sin perder la perspectiva de los resultados de la Resonancia Magnética Nuclear, ambas metodologías combinadas resultan de gran utilidad, para asegurar la calidad de los productos que se encuentran en el mercado, todo para el beneficio de los usuarios. De los detalles que se lograron observar es que muchos de los productos fabricados localmente no contienen los principios activos que se supone que tienen, y esto puede deberse al control deficiente por parte de las autoridades regulatorias.

\section{Referencias}

[1] W. Andlauer and P. Fürst, "Nutraceuticals: a piece of history, present status and outlook," Food Res. Int. 35, pp. 171-176, 2002.

[2] M. Intelligence, "NUTRACEUTICALS MARKET - GROWTH, TRENDS, AND FORECAST (2019 - 2024)," [Online]. Available: https://www.mordorintelligence.com/industry-reports/global-nutraceuticals-market-industry. [Accessed 138 2019].

[3] K. S. Bishop, C. Kao, J. Xu, M. Glucina, R. Paterson and L. Ferguson, "From 2,000 years of Ganoderma lucidum to recent developments in," Phytochemistry 114, pp. 56-65, 2015.

[4] F. Shahidi, "Nutraceuticals and functional foods: whole versus processed foods," Trends Food Sci. Technol. 20, pp. 376-387, 2009.

[5] H. Schilcher, S. Kammerer and T. Wegener, "Grundlegendes zur rationalen Phytotherapie," in Leitfaden Phytotherapie, Jena, Elsevier, Urban \& Fischer, 2007, pp. 1-30.

[6] A. Smith, S. Jogalekar and A. Gibson, "Regulation of natural health products in Canada," Regulation of natural health products in, p. 507-510, 2014.

[7] P. Coppens, M. da Silva and S. Pettman, "European regulations on nutraceuticals, dietary supplements, and functional foods: a framework based on safety," Toxicology 221, p. 59-74, 2006.

[8] S. C. d. I. Jurídica, "http://www.pgrweb.go.cr," [Online]. Available: http://www.pgrweb.go.cr/scij/Busqueda/ Normativa/Normas/nrm_texto_completo.aspx?param1=NRTC\&nValor1=1\&nValor2=75604\&nValor3=97190\&p aram2=1\&strTipM=TC\&IResultado=1\&strSim=simp. [Accessed 208 2019].

[9] K. Wang, H. Zhang, L. Shen, Q. Du and J. Li, "Rapid separation and characterization of active flavonolignans of Silybum marianum by ultra-performance liquid chromatography coupled with electrospray tandem mass spectrometry," Journal of Pharmaceutical and Biomedical Analysis 53, p. 1053-1057, 2010.

[10] A. P. Minoja and C. Napoli, "NMR screening in the quality control of food and nutraceuticals," Food Research International, vol. 63, no. Part B, pp. 126-131, 2014.

[11] N. López-Gutiérrez, R. Romero-González, ,. J. Martínez Vidal and A. Garrido Frenich, "Quality control evaluation of nutraceutical products from Ginkgo biloba sing liquid chromatography coupled to high resolution mass spectrometry," Journal of Pharmaceutical and Biomedical Analysis 121, pp. 151-160, 2016.

[12] P. Mai Le, C. Milande, E. Martineau, P. Giraudeau and J. Farjon, "Quantification of natural products in herbal supplements: A combined NMR approach applied on goldenseal," Journal of Pharmaceutical and Biomedical Analysis, vol. 165, pp. 155-161, 2019.

[13] H.-Y. Wang and Y.-Q. Zhang, "The main active constituents and detoxification process of Ginkgo biloba seeds and their potential use in functional health foods," Journal of Food Composition and Analysis, vol. 83, p. Article 103247, 2019.

[14] P. Braquet and D. Hosford, "Ethnopharmacology and the development of natural PAF antagonists as therapeutic agents," J Ethnopharmacol 32, pp. 122-123, 1994. 
[15] L. Xiaoyan, L. Chen, T. Liu, H. Ke, X. Gong, Q. Wang, J. Zhang and X. Fan, "Chemical analysis, pharmacological activity and process optimization of the proportion of bilobalide and ginkgolides in Ginkgo biloba extract," Journal of Pharmaceutical and Biomedical Analysis, vol. 160, pp. 46-54, 2018.

[16] B. A. Pendry, V. Kemp, M. Hughes, J. Freeman, H. Nuhu, A. Sanchez-Medina, O. Corcoran and E. Galante, "Silymarin content in Silybum marianum extracts as a biomarker for the quality of commercial tinctures," Journal of Herbal Medicine, vol. 10, pp. 31-36, 2017.

[17] R. Gazák, D. Walterová and V. Kren, "Silybin and silymarin-new and emerging applications in medicine.," Curr Med Chem. 14(3), pp. 315-38., 2007.

[18] R. Bruni, V. Brighenti, L. K. Caesar, D. Bertelli, N. B. Cech and F. Pellati, "Analytical methods for the study of bioactive compounds from medicinally used Echinacea species," Journal of Pharmaceutical and Biomedical Analysis, vol. 160, pp. 443-477, 2018.

[19] J. Barnes, L. Anderson and J. Phillipson, "St John's wort (Hypericum perforatum L.): a review of its chemistry, pharmacology and clinical properties," J Pharm Pharmac, pp. 53, 583-600, 2001.

[20] D. Todd, T. Gulledge, E. Britton, M. Oberhofer, M. Leyte-Lugo, A. Moody, T. Shymanovich, L. Grubbs, M. Juzumaite, T. Graf, N. Oberlies, S. Faeth, S. Laster and N. Cech, "Ethanolic Echinacea purpurea Extracts Contain a Mixture of Cytokine-Suppressive and Cytokine-Inducing Compounds, Including Some That Originate from Endophytic Bacteria," PLOS ONE 10(5), p. e0124276, 2015.

[21] K. A. McKeown, "A review of the taxonomy of the genus Echinacea," in Perspectives on new crops and new uses, Alexandria, VA, ASHS Press, 1999, p. 482-489.

[22] E. Tatsis, V. Exarchou, A. Troganis and I. Gerothanassis, "1H NMR determination of hypericin and pseudohypericin in complex natural mixtures by the use of strongly deshielded $\mathrm{OH}$ groups," analytica chimica acta 607 , p. 219-226, 2008.

[23] J. Napolitano, T. Gödecke, M. Rodríguez-Brasco, B. Jaki, S. Chen, D. Lankin and G. Pauli, "The Tandem of Full Spin Analysis and qHNMR for the Quality Control of Botanicals Exemplified with Ginkgo biloba," J. Nat. Prod. 75, p. 238-248, 2012. 